\title{
Extending Postgrafting Cyclosporine Decreases the Risk of Severe Graft-versus-Host Disease after Nonmyeloablative Hematopoietic Cell Transplantation
}

\author{
Lauri Burroughs, ${ }^{1,2}$ Marco Mielcarek, ${ }^{1,3}$ Wendy Leisenring, ${ }^{1,4,5}$ Brenda M. Sandmaier, ${ }^{1,3}$ \\ David G. Maloney, ${ }^{1,3}$ Frédéric Baron, ${ }^{1}$ Paul J. Martin, ${ }^{1,3}$ Mary E.D. Flowers, ${ }^{1,3}$ Stephen J. Forman, ${ }^{6}$ \\ Thomas R. Chauncey, ${ }^{1,3,7}$ Benedetto Bruno, ${ }^{8}$ and Rainer Storb ${ }^{1,3,9}$
}

\begin{abstract}
Background. It is unknown whether the duration of systemic immunosuppressive treatment after allogeneic nonmyeloablative hematopoietic cell transplantation (HCT) might influence the incidence, severity, timing, and/or corticosteroid-responsiveness of graft-versus-host disease (GVHD).

Methods. We retrospectively analyzed outcomes among 185 patients with hematologic malignancies who were given grafts from HLA-matched related donors following conditioning with 2 Gy total body irradiation alone or in combination with fludarabine between December 1998 and March 2003. Postgrafting immunosuppression consisted of mycophenolate mofetil (days 0-27) in combination with 3 different cyclosporine (CSP) regimens: taper from (A) days 35 to $56(n=107),(B)$ days 56 to $77(n=35)$, and $(C)$ days 56 to $180(n=43)$.

Results. The overall incidences of grades II-IV and III-IV acute GVHD, and extensive chronic GVHD were 52\%, 13\%, and $56 \%$, respectively. The duration of CSP prophylaxis did not significantly influence the overall rate of acute GVHD (grade II-IV), extensive chronic GVHD, or non-relapse mortality. However, prolonged administration of CSP (group C) was associated with a significantly decreased hazard of grades III-IV acute GVHD (HR 0.2, 95\% CI [0.04, 0.9]) and with an increased likelihood of discontinuing all systemic immunosuppression (HR 2.4, 95\% CI [1.1, 5.2]) when compared to the shortest course of CSP (group A).

Conclusion. Longer CSP duration decreased the risk of severe GVHD and increased the likelihood of discontinuing all systemic immunosuppression after nonmyeloablative HCT with HLA-matched related grafts.
\end{abstract}

Keywords: Graft-versus-host disease, Nonmyeloablative conditioning, Allogeneic hematopoietic cell transplantation, Cyclosporine.

(Transplantation 2006;81: 818-825)

$\mathrm{T}$ he development of minimally toxic nonmyeloablative preparative regimens for allogeneic hematopoietic cell transplantation (HCT) has expanded the available treatment

\footnotetext{
L.B. and M.M. contributed equally to this study.

This work was supported in part by grants CA78902, CA18029, CA15704, HL36444, DK064715 and K12 CA076930 from the National Institutes of Health, Bethesda, MD. B.B. was supported by a grant from Ministero dell'Istruzione, dell'Università, della Ricerca (M.I.U.R.), Italy.

${ }^{1}$ Clinical Research Division, Fred Hutchinson Cancer Research Center, Seattle, WA.

${ }^{2}$ Department of Pediatrics, University of Washington, Seattle, WA.

${ }^{3}$ Department of Medicine, University of Washington, Seattle, WA.

${ }^{4}$ Public Health Sciences Division, Fred Hutchinson Cancer Research Center, Seattle, WA.

${ }^{5}$ Department of Biostatistics, University of Washington, Seattle, WA.

${ }^{6}$ City of Hope National Medical Center, Duarte, CA.

${ }^{7}$ VA Puget Sound Health Care System, Seattle, WA.

${ }^{8}$ University of Torino, Torino, Italy.

${ }^{9}$ Address correspondence to: Rainer Storb, M.D., Fred Hutchinson Cancer Research Center, 1100 Fairview Avenue N., D1-100, PO Box 19024, Seattle, WA 98109-1024.

E-mail: rstorb@fhcrc.org

Received 29 September 2005. Revision requested 19 October 2005.

Accepted 28 December 2005.

Copyright $\odot 2006$ by Lippincott Williams \& Wilkins

ISSN 0041-1337/06/8106-818

DOI: $10.1097 / 01 . t p .0000203556 .06145 .5 b$
}

options for patients who, due to older age or comorbid conditions, would otherwise not be candidates for conventional HCT. Compared to conventional HCT, nonmyeloablative regimens are mainly immunosuppressive and aimed at preventing immunologic graft rejection, while tumor eradication is mediated by graft-versus-tumor (GVT) effects elicited by minor histocompatibility antigen ( $\mathrm{mHA}$ ) disparities between donors and recipients.

Regimen-related toxicities and early mortality after HCT are substantially lower following nonablative compared with conventional conditioning $(1,2)$. In addition, previous studies have shown that the cumulative incidence of grade II-IV acute graft-versus-host disease (GVHD) after nonablative HCT was lower than after myeloablative HCT (3). Nevertheless, GVHD has remained a problem. Even though graftversus-host $(\mathrm{GVH})$ reactions are important for eradication of underlying malignancies, it is unclear what duration and severity of clinical GVHD are required for nonablative transplants to be successful. Different immunosuppressive drug combinations have been evaluated in efforts to decrease the incidence and severity of GVHD (4-17). However, the optimal duration of immunosuppressive therapy and the most effective drug combination to protect against GVHD have not been defined. 
Based on results of preclinical studies in a canine transplant model (18), we have developed a clinical nonmyeloablative preparative regimen consisting of low-dose total body irradiation (TBI; 2 Gy at $7 \mathrm{cGy} / \mathrm{min}$ on day 0 ) with or without fludarabine, followed by G-CSF-mobilized peripheral blood mononuclear cell (G-PBMC) infusions and postgrafting immunosuppression with mycophenolate mofetil (MMF) and cyclosporine (CSP). This regimen has been proven to be minimally toxic and well tolerated when used in patients with malignant and non-malignant hematologic diseases who were ineligible for conventional HCT (19-23).

Owing to the evolution of nonablative transplant protocols during the past 7 years, the duration of CSP administration in recipients of HLA-matched related grafts has been extended from 56 to 77 or 180 days, while the duration of MMF (days 0 to 27) was kept constant. In the present study we retrospectively analyzed whether the duration of postgrafting CSP influenced the incidence, severity, timing and/or corticosteroid-responsiveness of GVHD.

\section{PATIENTS AND METHODS}

One hundred eighty-five consecutive patients with hematologic malignancies who had HCT after nonmyeloablative conditioning from HLA-matched related donors were included in this analysis. Sixteen additional patients were excluded from the intention-to-treat analysis because the duration of CSP prophylaxis was not specified at the time of transplantation. Transplants were performed between December 1998 and March 2003 at the Fred Hutchison Cancer Research Center (FHCRC, $n=129$ ), City of Hope National Medical Center $(n=28)$, VA Puget Sound Health Care System $(n=20)$, and University of Torino $(n=8)$. Results were analyzed with data available as of December 16, 2003. Patients signed forms approved by the individual institutions' Institutional Review Boards documenting informed consent to participate in the clinical trials. All transplants were from related donors who were serologically matched for HLA-A, -B, and $-\mathrm{C}$, and allele level matched for HLA-DRB1 and -DQB1. Thirty-seven $(20 \%)$ of the 185 patients had failed autologous transplantation a median of 21 (range, 5-127) months before allogeneic transplantation.

\section{Preparative Regimens}

Patients were enrolled on 9 protocols that used 2 different conditioning regimens: 2 Gy TBI alone ( 7 cGy/min; day 0; $\mathrm{n}=67)$ or 2 Gy TBI plus fludarabine $\left(30 \mathrm{mg} / \mathrm{m}^{2} /\right.$ day; days -4 to $-2 ; n=118)$. Fifty-six of the 185 patients $(30 \%)$ received planned cytoreductive autologous transplants before the nonmyeloablative allografts.

\section{Immunosuppression after Transplant}

All patients received MMF $15 \mathrm{mg} / \mathrm{kg}$ orally every $12 \mathrm{hr}$ from days $0-27$ and CSP $6.25 \mathrm{mg} / \mathrm{kg}$ orally or $2.5 \mathrm{mg} / \mathrm{kg}$ intravenously every $12 \mathrm{hr}$ starting at day -3 or -1 , respectively. Owing to the evolution of nonablative transplant protocols, the duration of CSP prophylaxis varied. In the initial protocols, all patients received CSP through day 35 with taper to day 56. In an attempt at decreasing the observed incidence of GVHD, the duration of CSP was lengthened from 56 days to either 77 or 180 days in subsequent protocols. Patients were stratified to receive CSP through day 77 or 180 based on the perceived risk of recurrent or progressive disease. Patients with underlying malignancies deemed to be at high risk for recurrence were, in order to maximize GVT effects, prescribed faster CSP tapers (day 56 with taper until day 77) compared to those deemed to be at low risk for recurrence (day 56 with taper until day 180). We retrospectively compared outcomes of consecutive patients assigned to the following three CSP schedules: CSP at full doses until A) day 35 with taper until day $56(\mathrm{n}=107)$; B) day 56 with taper until day $77(n=35)$; C) day 56 with taper until day $180(n=43)$. In the remainder of this manuscript, they will be referred to as groups $\mathrm{A}, \mathrm{B}$, and $\mathrm{C}$, respectively. In addition to analyzing outcomes based on intention-to-treat, information regarding the actual administration of CSP, in particular, CSP taper initiation, discontinuation, and reasons for deviating from the prescribed CSP schedules were recorded.

\section{Disease Risk of Relapse}

Patients were retrospectively divided into three different groups (standard risk, high risk, and very high risk of relapse) based on their disease status at time of HCT. Standard risk included patients with acute myeloid or lymphoblastic leukemia in first complete remission (CR), myelodysplastic syndrome-refractory anemia, chronic myeloid leukemia (CML) in first chronic phase, chronic lymphocytic leukemia, low-grade non-Hodgkin lymphoma, high or intermediate grade non-Hodgkin lymphoma in CR, Hodgkin disease in CR, multiple myeloma in CR or with minimal residual disease. High risk included patients with CML in second chronic phase, myeloproliferative disorders, multiple myeloma, Hodgkin disease, and high or intermediate grade nonHodgkin lymphoma not in CR. Very high risk included patients with acute leukemia greater than second CR, CML in accelerated phase or blast crisis, and myelodysplastic syndrome-refractory anemia with excess blasts or in transformation.

\section{GVHD Grading and Treatment}

Diagnosis and clinical grading of acute and chronic GVHD were performed according to established criteria (24, 25). Treatment decisions were based on the attending physicians' assessment of the severity of GVHD. In most cases, biopsies were done to confirm clinical findings. The typical primary treatment of acute GVHD consisted of prednisone (1-2 mg/kg per day; taper started after 14 days), and CSP, $\mathrm{MMF}$, or both were usually resumed at full doses at the time prednisone treatment was indicated. Prednisone with or without CSP was also used for the primary treatment of extensive chronic GVHD. Information regarding the initiation and discontinuation of prednisone, and other systemic immunosuppressive agents was collected retrospectively. This information allowed determination of time of onset of both acute and chronic GVHD and served as a marker for therapyresponsiveness of GVHD.

\section{Modulation of Immunosuppression after Recurrence or Progression of Malignancy}

In patients with recurrent or progressive malignant disease without active GVHD who were still on systemic immunosuppressive therapy, immunosuppressive drugs 
were usually rapidly tapered to induce GVT effects. Patients' follow-ups were therefore truncated at the time the CSP dose was tapered early as a result of suspected disease relapse or progression. Therefore, our analysis did not include GVHD events that occurred following changes in the prescribed immunosuppression schedule due to relapse or progressive disease. Among 59 patients with disease relapse or progression, CSP was either tapered or discontinued earlier than prescribed in seven patients. Of these seven patients, two developed GVHD.

\section{Donor Lymphocyte Infusion (DLI)}

Twenty-two patients (12\%) were treated with DLI for disease relapse/progression or decreasing chimerism. GVHD and immunosuppressive therapy events occurring after DLI were not included in this analysis.

\section{Graft Rejection}

Five patients $(3 \%)$ rejected their grafts $(<5 \%$ donor CD3 + T-cell chimerism), and GVHD, immunosuppressive therapy outcomes, and progression/relapse events were not included in this analysis if they occurred after rejection.

\section{Statistical Analysis}

Demographic factors were summarized using percentages, medians, and range values. Statistical comparisons between factors were made using a Kruskal Wallis test for continuous outcomes and chi-square or Fisher's exact tests for categorical factors. Overall survival and progression-free survival were estimated by the Kaplan and Meier method. Cumulative incidence curves were used to estimate acute and extensive chronic GVHD, initiation of prednisone, discontinuation of all immunosuppressive therapy, and relapse and non-relapse mortality. Death, rejection, DLI, and relapse/disease progression were treated as competing risk events in these analyses, where appropriate for each endpoint. In addition, initiation of CSP taper or CSP discontinuation due to disease relapse/progression was treated as a competing risk event for GVHD and immunosuppressive therapy endpoints. Cumulative incidence of both grades II-IV and III-IV acute GVHD used time of first GVHD symptoms as the onset time. Cox proportional hazards models were used to evaluate univariate and multivariate effects of covariates on time to event outcomes. Possible confounding factors considered for inclusion in multivariate models were patient age at transplant, preparative regimen, primary disease risk group ('standard,' 'high,' and 'very high'), and the number of prior chemotherapy regimens. All factors were considered together in multivariable models, and those that did not markedly affect the hazard ratio for other factors and were not statistically significant themselves were eliminated. All $P$ values for Cox regression were derived from likelihood ratio statistics and were two-sided.

\section{RESULTS}

One hundred eighty-five patients with hematologic malignancies were included in this study. The median patient age was 55 (range, 18-73) years. Details regarding patient characteristics are provided in Table 1. In part owing to the evolution of transplant protocols, imbalances of patient characteristics between the three different CSP taper groups ex- isted with regards to the disease, preparative regimen, and disease risk.

\section{GVHD}

Among the 185 patients included in this analysis, the overall incidences of grades II-IV, III-IV acute GHVD, and extensive chronic GVHD were 52\%, 13\%, and 56\%, respectively. The cumulative incidences of grades II-IV and III-IV acute GVHD in CSP taper groups A, B, and C were $57 \%(n=61), 43 \%(n=15), 49 \%(n=21)$, and $15 \%(n=16)$, $20 \%(n=7), 5 \%(n=2)$, respectively. While the overall incidences of grades II-IV acute GVHD were similar among the three CSP taper groups $(P=0.76$; Figure $1 \mathrm{~A})$, there was a strong suggestion $(P=0.06)$ of a decreased hazard of grades III-IV acute GVHD detected among patients assigned to the longest CSP prophylaxis compared to those assigned to the shortest prophylaxis (group C vs. A; HR 0.3, 95\% CI [0.1, 1.3]). There was a significantly decreased hazard of grades III-IV acute GVHD detected among patients assigned to the longest CSP prophylaxis compared to those assigned to the intermediate duration (group C vs. B; HR 0.2, 95\% CI [0.04, 0.9 ], $P=0.02$; Figure $1 \mathrm{~B}$ ). After adjusting for patient age, preparative regimen, and disease risk in multivariate analysis, there was a significantly decreased hazard of grades III-IV acute GVHD among patients assigned to the longest CSP prophylaxis compared to those assigned to the shortest CSP prophylaxis regimen (group C vs. A; HR 0.2, 95\% CI [0.04, 0.9], $P=0.01$; Table 2). Adjusting for the number of prior chemotherapy regimens did not change this result (data not shown). Multivariate analysis also showed increased risks of grades III-IV acute GVHD among patients given fludarabine and TBI compared to those given TBI alone (HR 5.2, 95\% CI [0.7, 40.9]; $P=0.05$; Table 2). Although the day 28 donor T-cell chimerism was significantly different between groups A, B, and $\mathrm{C}$, the degree of donor $\mathrm{T}$-cell chimerism did not influence the risk of subsequent acute grades III-IV GVHD in univariate or multivariate analysis that adjusted for CSP taper group (data not shown). With a median follow-up of 29 (range, 6-55) months (38 (range 23-55), 13 (range, 7-30) and 13 (range, 6-30) respectively for groups A, B and C) and 106 patients (57\%) alive, there was a significantly decreased incidence of extensive chronic GVHD among patients assigned to receive CSP until day 180 compared to the shortest CSP duration (group C vs. A, HR 0.6, 95\% CI $[0.4,1.0] ; P=0.05$; Figure $1 \mathrm{C}$ ). However, after adjusting for patient age, preparative regimen, and disease risk in multivariate analysis, this difference was merely a trend (group C vs. A, HR 0.7, 95\% CI $[0.4,1.2] ; P=0.14)$.

Corticosteroids were the first-line therapy for patients who developed GVHD. Thus, prednisone initiation served as a marker for the onset of both acute and chronic GVHD that required systemic immunosuppression. The cumulative incidences for prednisone initiation were similar among patients in the three different CSP groups ( $P=0.38$; Figure 2$)$. Therefore, the intended CSP duration did not influence the time of onset of acute or chronic GHVD requiring therapy.

\section{Discontinuation of Systemic Immunosuppression}

Discontinuation of all systemic immunosuppressive agents served as a marker for successful GVHD prophylaxis 
TABLE 1. Patient characteristics

\begin{tabular}{|c|c|c|c|c|}
\hline & $\begin{array}{l}\text { CSP day 35, } \\
\text { taper to day } 56\end{array}$ & $\begin{array}{c}\text { CSP day 56, } \\
\text { taper to day } 77\end{array}$ & $\begin{array}{c}\text { CSP day } 56, \\
\text { taper to day } 180\end{array}$ & $P$ value ${ }^{a}$ \\
\hline $\mathrm{n}$ & 107 & 35 & 43 & \\
\hline Median age, years (range) & $53(18-73)$ & $56(26-73)$ & $57(36-71)$ & 0.35 \\
\hline Sex $\%$, female/male & $32 / 68$ & $37 / 63$ & $42 / 58$ & 0.49 \\
\hline Disease, $\mathrm{n}(\%)$ & & & & 0.02 \\
\hline CML & $7(7)$ & $0(0)$ & $0(0)$ & \\
\hline CLL & $9(8)$ & $1(3)$ & $8(19)$ & \\
\hline ALL/AML & $9(8)$ & $6(17)$ & $2(5)$ & \\
\hline MDS & $18(17)$ & $6(17)$ & $4(9)$ & \\
\hline NHL/HD & $27(25)$ & $17(49)$ & $13(30)$ & \\
\hline $\mathrm{MM}$ & $35(33)$ & $5(14)$ & $16(37)$ & \\
\hline Wald & $2(2)$ & $0(0)$ & $0(0)$ & \\
\hline Donor, n $(\%)$ & & & & 1.00 \\
\hline HLA matched sibling & $106(99)$ & $35(100)$ & $43(100)$ & \\
\hline HLA matched related (non-sibling) & $1(1)$ & $0(0)$ & $0(0)$ & \\
\hline Preparative regimens, $\mathrm{n}(\%)$ & & & & $<0.001$ \\
\hline TBI, 2 Gy & $24(22)$ & $0(0)$ & $0(0)$ & \\
\hline Flu + TBI, 2 Gy & $48(45)$ & $32(91)$ & $25(58)$ & \\
\hline Auto + Flu + TBI, 2 Gy & $1(1)$ & $3(9)$ & $9(21)$ & \\
\hline Auto + TBI, 2 Gy & $34(32)$ & $0(0)$ & $9(21)$ & \\
\hline Disease risk, $\mathrm{n}(\%)$ & & & & $<0.001$ \\
\hline Standard risk & $51(48)$ & $12(34)$ & $32(74)$ & \\
\hline High risk & $48(45)$ & $20(57)$ & $11(26)$ & \\
\hline Very high risk & $8(7)$ & $3(9)$ & $0(0)$ & \\
\hline DLI, n (\%) & $16(15)$ & $2(6)$ & $4(9)$ & 0.33 \\
\hline Rejection, n (\%) & $4(4)$ & $0(0)$ & $1(2)$ & 0.83 \\
\hline
\end{tabular}

${ }^{a}$ Two-sided $P$ values from Kruskal-Wallis test for age, chi-square or Fisher's exact test for all other factors.

ALL, acute lymphoblastic leukemia; AML, acute myelogenous leukemia; Auto, autologous; CLL, chronic lymphocytic leukemia; CML, chronic myeloid leukemia; DLI, donor lymphocyte infusion; FLU, fludarabine; HD, Hodgkin disease; MDS, myelodysplastic syndrome; MM, multiple myeloma; NHL, nonHodgkin lymphoma; TBI, total body irradiation; y, year; Wald, Waldenström's disease.

and/or responsiveness of GVHD to therapy. The cumulative incidences of discontinuation of all systemic immunosuppression by 24 months were $25 \%, 16 \%$, and $50 \%$ for groups $\mathrm{A}, \mathrm{B}$, and $\mathrm{C}$, respectively. There was a strong suggestion that time to discontinuation of all systemic immunosuppression was shorter for patients assigned to receiving CSP until day 180 (group C) compared to those assigned to receiving the shortest duration of CSP (group A, $P=0.06$; Figure 3 ). Differences between groups $\mathrm{B}$ and $\mathrm{C}$ were not statistically significant $(P=0.12)$. When included in a multivariable model that adjusted for age at transplant, preparative regimen, and disease risk, the association between longer duration of CSP exposure and the likelihood of earlier discontinuation of systemic immunosuppression became significant (group $\mathrm{C}$ vs. group $\mathrm{A} ; \mathrm{HR}=2.4,95 \% \mathrm{CI}[1.1,5.2], P=0.03)$. There was no significant difference in the time to discontinuation of all systemic immunosuppression according to the prescribed duration of CSP prophylaxis among patients who developed extensive chronic GVHD $(P=0.35)$.

\section{Nonrelapse Mortality}

Twenty-two of the 44 deaths from non-relapse causes were GVHD related. The cumulative incidences of non-relapse mortality (NRM) according to the prescribed duration of CSP prophylaxis were not significantly different $(P=0.32$; Figure 4). Adjusting for patient age, preparative regimen, and disease risk in multivariate analysis did not change this finding. However, there was an increased hazard of NRM among those patients who received fludarabine and TBI compared to TBI alone as part of their conditioning (HR 3.5; 95\% CI $[1.1,11.4] ; P=0.02)$.

\section{Risk of Recurrent Malignancy}

As would be expected, the hazards of progression or relapse were significantly higher for patients in the "high" and "very high" disease risk categories relative to "standard" risk patients (both $P<0.01$ ). In univariate analysis, we found a significantly decreased hazard of relapse among patients assigned to group $\mathrm{C}$ compared to those assigned to group $\mathrm{B}$ (HR $0.4 ; 95 \%$ CI $[0.2,1.0] ; P=0.04$ ). This could be explained by the fact that some transplant protocols used the perceived likelihood of recurrent or progressive malignancy to assign patients to a relatively faster or slower CSP taper. After adjusting for disease risk in multivariate analysis, however, there was no significant association between the assigned duration of prophylactic CSP and the risk of recurrent malignancy (HR 0.5 ; $95 \%$ CI $[0.2,1.3] ; P=0.12)$.

\section{Reason for Deviation from Prescribed CSP Taper Schedules}

The results reported thus far were based on "intentionto-treat." We sought to determine whether this type of analysis was a reasonable representation of the CSP regimen administered to patients. Possible reasons for deviating from the prescribed CSP regimen included GVHD, disease relapse or progression, CSP toxicity, and death prior to CSP taper 

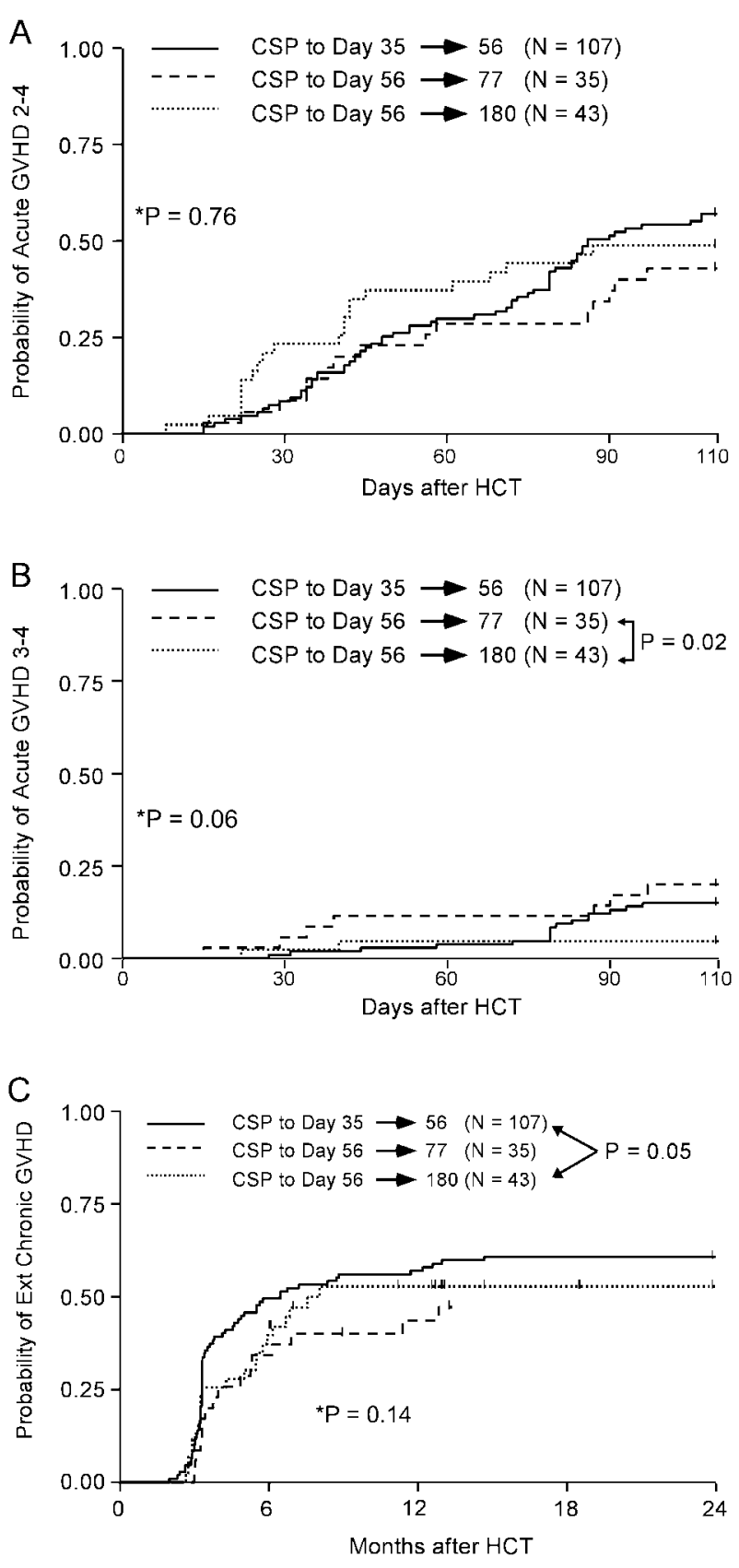

FIGURE 1. Intention-to-treat analysis. (A) Probability of acute GVHD (grades 2-4) according to duration of CSP prophylaxis. (B) Probability of severe acute GVHD (grades 3-4) according to duration of CSP prophylaxis. (C) Probability of extensive chronic GVHD according to duration of CSP prophylaxis. (Ext=extensive). ${ }^{*} P$ denotes value for overall comparison among the three groups.

initiation. Among the 185 patients in this study, CSP was tapered as originally prescribed per protocol in 103 patients (56\%). In 6 patients (4\%), the CSP taper was initiated early due to either recurrent or progressive malignancy $(n=3 ; 2 \%)$ or CSP toxicity $(n=3 ; 2 \%)$. Nine patients $(5 \%)$ died before the prescribed initiation of the CSP taper, and in 52 patients $(28 \%)$ the initiation of the CSP taper was delayed because of the development of GVHD. For 15 patients (8\%), the times of initiating or the reasons for delaying the CSP taper could not be determined. The proportions of patients who initiated the CSP taper according to respective transplant protocols were $64 \%, 46 \%$, and $44 \%$ for groups $\mathrm{A}, \mathrm{B}$, and $\mathrm{C}$, respectively $(P=0.04)$. However, in all three patient groups, the primary reason for not initiating the CSP taper according to protocol was the development of GVHD $(21 \%, 34 \%$, and $42 \%$ for groups $\mathrm{A}, \mathrm{B}$, and $\mathrm{C}$, respectively).

\section{Tempo of CSP Withdrawal and Risk of Severe GVHD}

To determine whether there was a possible association between the tempo of CSP withdrawal and the risk of GVHD, data from patients in groups $\mathrm{A}, \mathrm{B}$ and $\mathrm{C}$ who were alive without GVHD at day 56, were analyzed for the subsequent occurrence of GVHD. After adjusting for preparative regimen, age at transplant, and disease risk in multivariate Cox regression analysis, the tempo of CSP withdrawal in group A compared to C was a significant risk factor for grades II-IV GVHD $(P=0.01)$, however, was not a significant risk factor for grades II-IV acute GVHD in group B compared to C $(P=0.36)$. Due to the small number of events of grades III-IV acute GVHD after day 56 (13, 3 and 0 for groups A, B and C, respectively), a similar Cox regression analysis could not be performed. Instead, a univariate log rank test found a decreased risk of grade III-IV acute GVHD after day 56 among patients in group $\mathrm{C}$ compared to those in group $\mathrm{A}(P=0.03)$ and group $\mathrm{B}$ $(P=0.04)$.

\section{DISCUSSION}

The analysis showed that recipients of nonmyeloablative transplants from HLA-matched related donors who were assigned to a prolonged course of postgrafting CSP (180 days vs. 56 or 77 days), had a statistically significant decreased risk of severe (grades III-IV) acute GVHD and an increased likelihood of discontinuing systemic immunosuppressive therapy. The risk of developing extensive chronic GVHD was not affected by the prescribed duration of the CSP prophylaxis.

Even though GVH-reactions ensuing after nonmyeloablative HCT are, in theory, needed for achieving stable engraftment and for eradication of the underlying malignant diseases, the prevention of severe acute GVHD and its documented associated morbidity and mortality have remained important objectives (26-29). A recent study among recipients of HLA-matched related and unrelated nonmyeloablative transplants conducted at our institution demonstrated that grades III-IV acute GVHD resulted in significantly increased non-relapse mortality without measurable protective effects against recurrent malignancies. The protective effects against disease recurrence and the consequent superior progression-free survival were exclusively associated with extensive chronic GVHD (30). In light of these results, the prevention of (severe) acute GVHD appeared to be more desirable than the prevention of chronic GVHD. The current analysis suggested that the risk of severe acute GVHD could be minimized without affecting the risk of chronic GVHD by prescribing at least 2 months of CSP, followed by a taper over the subsequent 4 months. 
TABLE 2. Multivariate analysis of severe acute GVHD grades 3-4

\begin{tabular}{|c|c|c|c|c|}
\hline Variable & & $\begin{array}{c}\text { Adjusted } \\
\text { hazard ratio }\end{array}$ & $P$ value & $\begin{array}{c}95 \% \\
\text { confidence } \\
\text { interval }\end{array}$ \\
\hline \multirow{3}{*}{ CSP duration } & Day 35 , taper to day 56 & 1.0 & - & - \\
\hline & Day 56, taper to day 77 & 1.0 & 0.99 & $0.4,2.7$ \\
\hline & Day 56 , taper to day 180 & 0.2 & 0.01 & $0.04,0.9$ \\
\hline \multirow[t]{4}{*}{ Conditioning regimen } & TBI & 1.0 & - & - \\
\hline & $\mathrm{Flu}+\mathrm{TBI}$ & 5.2 & 0.05 & $0.7,40.9$ \\
\hline & Auto + Flu + TBI & 8.8 & 0.09 & $0.7,114.2$ \\
\hline & Auto + TBI & 2.9 & 0.29 & $0.3,24.6$ \\
\hline \multirow[t]{2}{*}{ Age (years) } & $<55$ & 1.0 & - & - \\
\hline & $\geq 55$ & 1.1 & 0.76 & $0.5,2.5$ \\
\hline \multirow[t]{3}{*}{ Disease risk } & Standard & 1.0 & - & - \\
\hline & High & 1.1 & 0.77 & $0.5,2.6$ \\
\hline & Very High & 0.4 & 0.40 & $0.1,3.6$ \\
\hline
\end{tabular}

Auto, autologous; FLU, fludarabine; TBI, total body irradiation.

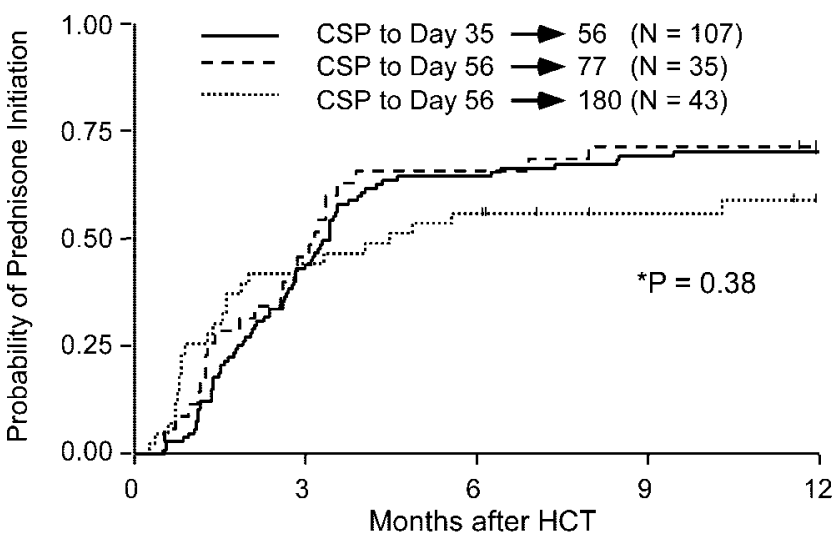

FIGURE 2. Probability of prednisone initiation according to duration of CSP prophylaxis. Intention-to-treat analysis. $* P$ denotes value for overall comparison among the three groups.

The initiation of prednisone therapy served as a marker for the onset of both acute and chronic GVHD. We did not find significant differences in either the times to onset of prednisone therapy nor in the cumulative incidences of prednisone initiation among patients assigned to the three different CSP groups. This suggested that maintenance of therapeutic CSP levels $(150-400 \mathrm{ng} / \mathrm{ml})$ until at least 2 months after transplant, followed by a "slow" CSP taper (6\% per week) did not affect the time of onset or overall incidence of grades II-IV acute GVHD but rather prevented progression of this complication to grades III-IV acute GVHD.

A systematic analysis of the effects of the duration of immunosuppressive prophylaxes on outcomes after nonmyeloablative HCT has not, to our knowledge, been performed. Two randomized studies compared 2 vs. 6 months (31), and 6 vs. 24 months (32) of CSP given after myeloablative HCT from HLA-identical siblings or HLA-matched unrelated donors. Both studies failed to find significant differences in the incidences of chronic GVHD and NRM among patients given the shorter compared to the longer CSP regimens. In contrast, two non-randomized studies among recipients of myeloablative transplants from HLA-identical siblings suggested

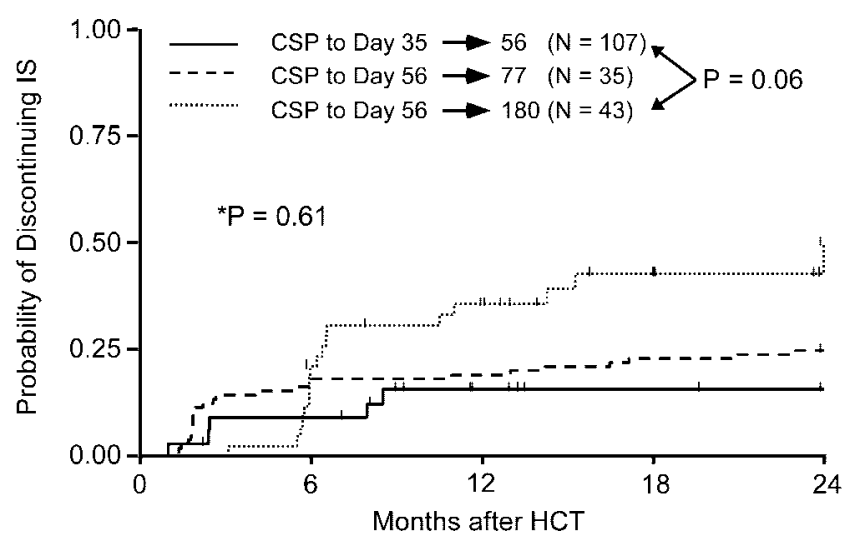

FIGURE 3. Probability of discontinuing all systemic immunosuppression according to duration of CSP prophylaxis. Intention-to-treat analysis. IS, immunosuppression. $* P$ denotes value for overall comparison among the three groups.

that prolonged CSP prophylaxis ( $>5$ months vs. $<5$ months; and 12 vs. 6 months) was associated with protection against chronic GVHD $(33,34)$. The impact of the duration of CSP prophylaxis on the incidence or severity of acute GVHD could not be addressed in previous studies because patients were on CSP prophylaxis at the time acute GVHD developed.

An important marker not only for the successful prevention of GVHD but also for its therapy responsiveness has been the discontinuation of all systemic immunosuppression. Multivariate analysis demonstrated a significantly increased likelihood of discontinuing all systemic immunosuppression among patients assigned to receiving a prolonged course of CSP (group C; intent to complete taper by day 180) as compared to those assigned to receive the shortest course of CSP (group A; intent to complete taper by day 56). Hence, the relatively slow withdrawal of CSP not only decreased the risk of severe acute GVHD but also appeared to facilitate the eventual development of donor/recipient tolerance as evidenced by the ability to discontinue all systemic immunosuppressive therapy.

A potential limitation of this study was the fact that 


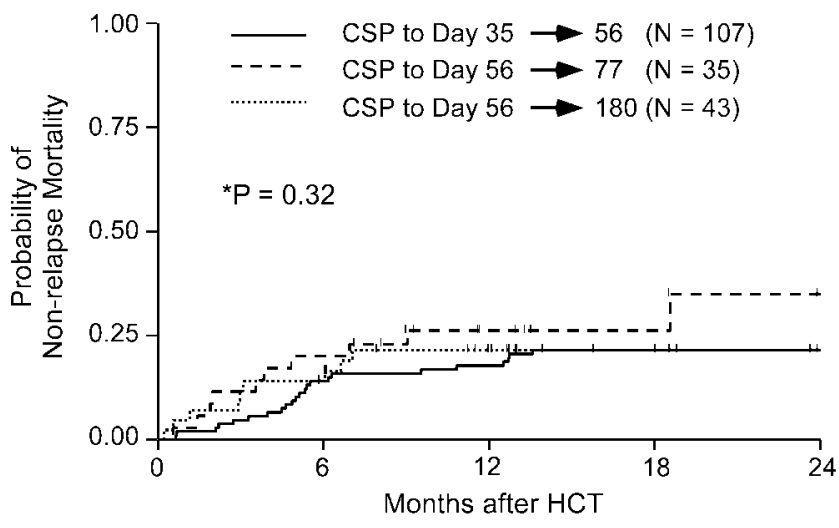

FIGURE 4. Probability of non-relapse mortality according to duration of CSP prophylaxis. Intention-to-treat analysis. $* P$ denotes value for overall comparison among the three groups.

patients were evaluated based on "intention-to-treat." However, an evaluation of incidence and severity of GVHD based on actual durations of CSP exposure would have led to biased results. For example, the primary reason patients remained on CSP longer than specified by respective protocols was the occurrence of GVHD. Therefore, an evaluation of the impact of actual CSP exposure on the risk of GVHD would have led to the circular conclusion that extending the CSP prophylaxis increased the risk of GVHD.

Since these were not concurrent protocols, it is possible that the differences in GVHD rates seen between protocol groups could be explained by treatment changes over time. Although we have attempted to adjust for specific factors known to have changed over time, such as the addition of 3 doses of fludarabine to the 2 Gy TBI in the conditioning regimen, it is possible that other factors changed that we did not consider.

To address the potential limitation of an analysis by intention-to-treat, we determined the incidence of GVHD occurring after day 56 in a landmark analysis including all patients in groups $\mathrm{A}, \mathrm{B}$ and $\mathrm{C}$ who were alive without GVHD at day 56. In spite of the relatively low number of events of grades III-IV GVHD occurring after day 56 among the patients in the 3 groups $(13,3$ and 0 for groups A, B and C, respectively), the analysis suggested that a slower CSP taper was associated with a protection against grades III-IV acute GVHD, although this result should be confirmed due to the small number of subjects available for analysis.

Finally, multivariate analysis adjusted for CSP exposure, patient age, and disease risk showed that patients conditioned with fludarabine and TBI compared to TBI alone experienced both higher incidences of grade III-IV acute GVHD and NRM. Fludarabine had been added to $2 \mathrm{~Gy}$ TBI in a successful attempt to overcome the $20 \%$ incidence of nonfatal graft rejections observed among the first 44 recipients of nonmyeloablative transplants from HLA-matched related donors after 2 Gy TBI alone (21). Subsequent analyses demonstrated a significantly decreased incidence of graft rejection in patients given fludarabine and TBI (3\%) compared to those given TBI alone $(P=0.001)$. While the risks of relapse were similar between the two groups, patients conditioned with fludarabine/TBI had a significantly increased NRM from infections compared to those given TBI only $(P=0.02)(22)$. Given that these findings were based on retrospective analyses of data from patients who were not transplanted concurrently, we are presently conducting a prospective, randomized, phase III study comparing conditioning with TBI versus fludarabine/TBI.

In summary, we showed that after nonablative HCT from HLA-matched related donors, a prescribed 2-month course of CSP followed by a 4-month taper decreased the risk of grades III-IV acute GVHD and increased the likelihood of discontinuing all systemic immunosuppression when compared to two shorter CSP regimens. Protection against severe acute GVHD did not affect the risks of chronic GVHD or relapse. Future prospective studies are needed to determine whether substituting alternative calcineurin inhibitors, such as tacrolimus, for CSP, or extending the use of antimetabolites, such as MMF, beyond one month after transplant might be more effective in preventing severe or therapy-refractory GVHD without compromising engraftment and control of the underlying malignancies.

\section{ACINOWLEDGIMENTS}

We thank Heather Hildebrant, Chris Davis and Deborah Bassuk for data management; the research nurses Steve Minor, Mary Hinds, and John Sedgwick; the nursing and clinical staff for their dedicated care of patients; and Bonnie Larson, Helen Crawford, and Sue Carbonneau for help with manuscript preparation.

\section{REFERENCES}

1. Sorror ML, Maris MB, Storer B, et al. Comparing morbidity and mortality of HLA-matched unrelated donor hematopoietic cell transplantation after nonmyeloablative and myeloablative conditioning: influence of pretransplant comorbidities. Blood 2004; 104: 961-968.

2. Diaconescu R, Flowers CR, Storer B, et al. Morbidity and mortality with nonmyeloablative compared to myeloablative conditioning before hematopoietic cell transplantation from HLA matched related donors. Blood 2004; 104: 1550-1558.

3. Mielcarek M, Martin PJ, Leisenring W, et al. Graft-versus-host disease after nonmyeloablative versus conventional hematopoietic stem cell transplantation. Blood 2003; 102: 756-762.

4. Ramsay NKC, Kersey JH, Robison LL, et al. A randomized study of the prevention of acute graft-versus-host disease. N Engl J Med 1982; 306: 392-397.

5. Storb R, Deeg HJ, Pepe M, et al. Graft-versus-host disease prevention by methotrexate combined with cyclosporin compared to methotrexate alone in patients given marrow grafts for severe aplastic anaemia: Long-term follow-up of a controlled trial. Br J Haematol 1989; 72: 567-572.

6. Storb R, Deeg HJ, Whitehead J, et al. Methotrexate and cyclosporine compared with cyclosporine alone for prophylaxis of acute graft versus host disease after marrow transplantation for leukemia. $N$ Engl J Med 1986; 314: 729-735.

7. Storb R, Deeg HJ, Pepe M, et al. Methotrexate and cyclosporine versus cyclosporine alone for prophylaxis of graft-versus-host disease in patients given HLA-identical marrow grafts for leukemia: Long-term follow-up of a controlled trial. Blood 1989; 73: 1729-1734.

8. Forman SJ, Blume KG, Krance RA, et al. A prospective randomized study of acute graft-v-host disease in 107 patients with leukemia: Methotrexate/prednisone v cyclosporine A/prednisone. Transplant Proc 1987; 19: 2605-2607.

9. Santos GW, Tutschka PJ, Brookmeyer R, et al. Cyclosporine plus methylprednisolone versus cyclophosphamide plus methylprednisolone as prophylaxis for graft-versus-host disease: A randomized double-blind study in patients undergoing allogeneic marrow transplantation. Clin Transplant 1987; 1: 21-28. 
10. Storb R, Pepe M, Anasetti C, et al. What role for prednisone in prevention of acute graft-versus-host disease in patients undergoing marrow transplants? Blood 1990; 76: 1037-1045.

11. Chao NJ, Schmidt GM, Niland JC, et al. Cyclosporine, methotrexate, and prednisone compared with cyclosporine and prednisone for prophylaxis of acute graft-versus-host disease. N Engl J Med 1993; 329: 1225-1230.

12. Deeg HJ, Lin D, Leisenring W, et al. Cyclosporine or cyclosporine plus methylprednisolone for prophylaxis of graft-versus-host disease: a prospective, randomized trial. Blood 1997; 89: 3880-3887.

13. Ratanatharathorn V, Nash RA, Przepiorka D, et al. Phase III study comparing methotrexate and tacrolimus (Prograf, FK506) with methotrexate and cyclosporine for graft-versus-host-disease prophylaxis after HLA-identical sibling bone marrow transplantation. Blood 1998; 92 : 2303-2314.

14. Ruutu T, Volin L, Parkkali T, et al. Cyclosporine, methotrexate, and methylprednisolone compared with cyclosporine and methotrexate for the prevention of graft-versus-host disease in bone marrow transplantation from HLA-identical sibling donor: a prospective randomized study. Blood 2000; 96: 2391-2398.

15. Locatelli F, Bruno B, Zecca M, et al. Cyclosporin A and short-term methotrexate versus cyclosporin A as graft versus host disease prophylaxis in patients with severe aplastic anemia given allogeneic bone marrow transplantation from an HLA-identical sibling: results of a GITMO/EBMT randomized trial. Blood 2000; 96: 1690-1697.

16. Nash RA, Antin JH, Karanes C, et al. Phase 3 study comparing methotrexate and tacrolimus with methotrexate and cyclosporine for prophylaxis of acute graft-versus-host disease after marrow transplantation from unrelated donors. Blood 2000; 96: 2062-2068.

17. Srinivasan R, Geller N, Chakrabarti S, et al. Evaluation of three different cyclosporine-based graft versus host disease (GVHD) prophylaxis regimens following nonmyeloablative hematopoietic stem cell transplantation (NST). Blood 2004; 104: 349a.

18. Storb R, Yu C, Wagner JL, et al. Stable mixed hematopoietic chimerism in DLA-identical littermate dogs given sublethal total body irradiation before and pharmacological immunosuppression after marrow transplantation. Blood 1997; 89: 3048-3054.

19. Maris MB, Niederwieser D, Sandmaier BM, et al. HLA-matched unrelated donor hematopoietic cell transplantation after nonmyeloablative conditioning for patients with hematologic malignancies. Blood 2003; 102: 2021-2030.

20. Niederwieser D, Maris M, Shizuru JA, et al. Low-dose total body irradiation (TBI) and fludarabine followed by hematopoietic cell transplantation (HCT) from HLA-matched or mismatched unrelated donors and postgrafting immunosuppression with cyclosporine and mycophenolate mofetil (MMF) can induce durable complete chimerism and sustained remissions in patients with hematological diseases. Blood 2003; 101: 1620-1629.

21. McSweeney PA, Niederwieser D, Shizuru JA, et al. Hematopoietic cell transplantation in older patients with hematologic malignancies: replacing high-dose cytotoxic therapy with graft-versus-tumor effects. Blood 2001; 97: 3390-3400.

22. Sandmaier BM, Maloney DG, Gooley TA, et al. Low dose TBI conditioning for hematopoietic stem cell transplants (HSCT) from HLAmatched related donors for patients with hematologic malignancies: influence of fludarabine or cytoreductive autografts on outcome. Blood 2002; 100: 145a.

23. Sandmaier BM, Maris M, Maloney DG, et al. Low-dose total body irradiation (TBI) conditioning for hematopoietic cell transplants (HCT) from HLA-matched related (MRD) and unrelated (URD) donors for patients with hematologic malignancies: a five-year experience. Blood 2003; 102: 78a.

24. Przepiorka D, Weisdorf D, Martin P, et al. 1994 Consensus conference on acute GVHD grading. Bone Marrow Transplant 1995; 15: 825-828.

25. Sullivan KM, Agura E, Anasetti C, et al. Chronic graft-versus-host disease and other late complications of bone marrow transplantation. Semin Hematol 1991; 28: 250-259.

26. Nash RA, Pepe MS, Storb R, et al. Acute graft-versus-host disease: analysis of risk factors after allogeneic marrow transplantation and prophylaxis with cyclosporine and methotrexate. Blood 1992; 80: 1838-1845.

27. Weiden PL, Flournoy N, Thomas ED, et al. Antileukemic effect of graftversus-host disease in human recipients of allogeneic-marrow grafts. N Engl J Med 1979; 300: 1068-1073.

28. Storb R, Prentice RL, Buckner CD, et al. Graft-versus-host disease and survival in patients with aplastic anemia treated by marrow grafts from HLA-identical siblings. Beneficial effect of a protective environment. N Engl J Med 1983; 308: 302-307.

29. Glucksberg H, Storb R, Fefer A, et al. Clinical manifestations of graftversus-host disease in human recipients of marrow from HL-Amatched sibling donors. Transplantation 1974; 18: 295-304.

30. Baron F, Maris MB, Sandmaier BM, et al. Graft-versus-tumor effects after allogeneic hematopoietic cell transplantation with nonmyeloablative conditioning. J Clin Oncol 2005; 23: 1993-2003.

31. Storb R, Leisenring W, Anasetti C, et al. Methotrexate and cyclosporine for graft-vs.-host disease prevention: what length of therapy with cyclosporine? Biol Blood and Marrow Transplantation 1997; 3: 194201.

32. Kansu E, Gooley T, Flowers MED, et al. Administration of cyclosporine for 24 months compared with 6 months for prevention of chronic graft-versus-host disease: a prospective randomized clinical trial (Brief Report). Blood 2001; 98: 3868-3870.

33. Bacigalupo A, Maiolino A, Van Lint MT, et al. Cyclosporin A and chronic graft versus host disease. Bone Marrow Transplant 1990; 6: 341-344.

34. Mengarelli A, Iori AP, Romano A, et al. One-year cyclosporine prophylaxis reduces the risk of developing extensive chronic graft-versus-host disease after allogeneic peripheral blood stem cell transplantation. Haematologica 2003; 88: 315-323. 